\title{
Development of Dyeing Mechanism of Wood with Reactive Dyes
}

\author{
Shengfei $\mathrm{Yu}^{1, \mathrm{a}}$, Dan $\mathrm{Xu}^{1, \mathrm{~b}}$, Xiao Zhang ${ }^{1, \mathrm{c}}$, Tianhui Yu${ }^{1, \mathrm{~d}}$, Yuan Liu ${ }^{1, \mathrm{e}}, \mathrm{Xu}$ Wang ${ }^{1, \mathrm{f}}$, \\ $\mathrm{Na} \mathrm{Li} i^{1, g}$ \\ ayushengfei503@sina.com, ${ }^{b}$ 842078405@qq.com, ${ }^{\circ}$ 1016628051@qq.com, ${ }^{\text {d9 } 949129352 @ q q . c o m, ~}$ \\ ${ }^{\mathrm{e} l i u y u a n 601220 @ 163 . c o m},{ }^{1}$ 120129275@qq.com, ${ }^{9}$ 478343978@qq.com,
}

Key words: reactive dyes; wood; permeability; dyeing mechanism

Abstract: The Transmission Theoretical Model of reactive dyes fluid in wood are systemically reviewed in the paper. It also are summarized the dyeing dynamics and thermodynamics and the reaction mechanism of reactive dye liquor with wood. The existing shortages and development direction of dyeing mechanism of wood with reactive dyes are also presented.

Wood is a kind of nature porous material with adsorbability and permeability. When dyeing, reactive dyes solution firstly wet, diffuse, adsorb on the wood surface, next permeate into the wood, then react with the hydroxy and the amino in wood fiber, finally fix in the wood. Dyeing effects are affected by the structure of reactive dyes, the structure of wood and dyeing craft[1]. Moreover, the dyeing mechanism can guide and regulate the dyeing craft. This paper summarizes the findings of Transmission Theoretical Model of reactive dyes fluid in wood, the dyeing dynamics and thermodynamics and the reaction mechanism of reactive dye liquor with wood.

\section{Transmission Theoretical Model of Reactive Dyes Fluid in Wood}

The dyeing solution entering into wood interior with diverse anisotropy in alternating direction depends on several transmission modes. There are two major transmission mode: permeation and diffusion[2]. The process that fluid flows through correlative holes inside the wood under the impact of static pressure gradient or capillary pressure gradient is called permeation. At present there are many research findings about permeation model, which are mostly based on Darcy law. The main models include Uniform parallel capillary model, Sebatian soft wood model, Bramhall wood longitudinal permeability effective section attenuation model, Petty model, Comstock soft wood model, Soft wood longitudinal gas penetration three-dimensional flow resistance network model[3], etc. The above models are mainly consider soft wood, gas as the main fluid medium, and these models are too complicated or too simplified about wood structure or having not considering reality enough, making the calculation results far from the actual one, so it is difficult to be accepted by engineering application. As to the hardwood with complex cellular structure and Wood liquid penetrant theory, they are not able to establish accurate models from its internal movement mechanism and heat transmission and mass transmission properties. Based on the great influence of wood structure on fluid permeability, wood is generally pretreated and mathematical model of wood extract extraction volume and pretreatment process parameters have been build up, thus not only avoiding the problem of accurately determining permeating law, but also making it easier to control the permeation process.

When the moisture content of the wood saturates, dyeing solution diffuses into wood under concentration gradient. The diffusion process complys with Fick diffusion law and the diffusion rate mainly depends on diffusion coefficient. The diffusion models include Diffusion coefficient measurement on carrier drift rate model[4], Alkali impregnating diffusion -reaction model[5], shrinking core model[6], among which Alkali impregnating model[7] can well predicts the concentration of lye of wood internal parts under different impregnating temperature, sequentially predicting the impregnation endpoint when the dyes reach the high adsorption rate.

\section{The Research on Dyeing Dynamics and Thermodynamics}

Researching dyeing kinetics and thermodynamics, we can master the dyeing mechanism, identify the dye property, optimize dyeing draft, eventually achieve the improvement of dyeing quality, 
reduction of energy consumption and production costs. The research mainly focuses on the reactive dyes dyeing textile fiber, the study of wood fiber is less. Jiang X.J. et al found that the adsorption of reactive dyes on cotton fiber under acid condition without salt is in line with the Langmuir adsorption model, and exothermic, the diffusion rate gradually reduce[8]. Li M.H. found that the dyeing dynamics of cotton fiber with reactive red 3BS and reactive blue KN-G in water bath or D5 suspension system both conform the quasi-second level model, half dyeing time decreased significantly, the adsorption of the two dyes in different systems is respectively accord with the Langmuir and Freundlich isothermal adsorption type[9]. The research results of Sui S.Y. et al. show that the diffusion coefficient of reactive dyes into bamboo fiber is greater than the cotton fiber and viscose fiber, and decreases with the increase of the dyeing time, increases with the increase of temperature [10]. Wang Y.G. et al studied on the performance of fluid horizontal transmission of mountain Yang with the alkali soaking, and quantitatively measured transverse diffusion coefficient under steady state conditions[11]. These achievements are based on the experimental data without establishing a mathematical model. In recent years, the scholars strengthen the research on mathematical model of various dyes dyeing various fiber, making every effort to get reliable dyeing dynamics model to correctly describe the dyeing process on fiber. The classical equations of dyeing kinetics are all established based on Fick's second law, such as the Me Bain equation, Hill equation, Vicerstaff equation, Grank equation and the equation of Frensdorff [12], most of these models assume that diffusion area and diffusion coefficient are both a certain value, which is not fit into the actual dyeing process.

\section{The Reaction Mechanism of Reactive Dyes Liquor with Wood.}

The main compounds of wood, cellulose, hemicellulose and lignin, whose molecular structure contains a mass of $-\mathrm{OH}$, are all possible to have nucleophilic substitution reaction or nucleophilic addition reaction with the reactive group of reactive dye and fix on the wood. Li J.L. found that reactive dyes can react with the cellulose, hemicellulose and lignin of wood to a covalent bond by FTIR and TG[13]. FTIR analysis of Lv X.H. shows the quaternary ammonium salt can promote the double bond between reactive dyes and acetyl of cellulose and hemicellulose, SEM analysis shows the granular size of reaction product is about $1 \mathrm{pm}$, which can enter into the cell wall through the pit in tube wall of wood[14].

Since the dyeing process is generally done in alkaline solution, reactive dyes have hydrolysis reaction inevitably besides reacting with wood fiber. Yu H.H.[15] conformed the hydrolysis products of three kinds of typed-M reactive dyes all contains $\beta$-Hydroxyl-Ethyl Sulphone, hydroxyethyl sulfone and vinyl sulfone by HPLC and HPLC - MS analysis techniques, and with increasing temperature or $\mathrm{pH}$, the hydrolysis rates of $\beta$-Hydroxyl-Ethyl Sulphone and vinyl sulfone significantly accelerate. Wang Z.J. et al [16] studied by RP-HPLC Ion method the hydrolysis reaction of the reactive brilliant blue $\mathrm{KN}-\mathrm{R}$ is quasi first level when the $[\mathrm{OH}-]$ is constant, hydrolysis rate increased significantly with the increase of temperature. The research results of Zhang R. et al[17] by HPLC show that $\mathrm{pH}$ value is the main factor affecting C.I. reactive blue 19 dye hydrolysis: dyes mainly stay in the form of sulfate or vinyl sulfone fundamentally without hydrolysis reaction When $\mathrm{pH}<11$, hydrolysis starts to happen and the hydrolysis rate increases with the increase of $\mathrm{pH}$ when $\mathrm{pH}>11$. Owoyomi O. et al[18] did the kinetic test of Alkaline hydrolysis of bright green triphenylmethane dyes using TTAB or TTPPBr or their compounds as a catalyst. They found that the area brilliant Green dye bounds with micelle surface is bigger in the pure TTAB than in the pure TTPPBr, yet sharply lowering in mixture. The binding constant sharply decreased with the increasing mole ratio of TTAB in the mixture.

\section{Existing Problems and Prospects}

After dyeing with reactive dyes, the color of wood becomes rich, the color fastness and light fastness becomes excellent and the dyeing staining depth becomes deeper. At the moment the dyeing mechanism has not been systematically researched, which makes industrial circles lack of 
scientific theoretical guidance when dyeing wood with reactive dyes. There are defects such as poor permeability and colourability, low color fastness. Dyeing techniques with practical popularization value have not been able to be developed. In future, wood reactive dyeing mechanism can mainly be developed from the following several aspects.

1) The structural characteristics of wood determine the permeation of reactive dyes in wood. The structure of wood can be improved by dyeing pretreatment (especially chemical reagents pretreatment). There is no established relationship between the extract of chemical pretreatment and pretreatment technological parameter, namely dissolution kinetics equation. The wood extract dissolution laws should be mastered, so that the permeation of the reactive dyes within the wood is much easier to implement.

2) The structure of the reactive dye affects the dyeing speed, depth, color, fastness, uniformity and other dyeing effect. The reactive dyes used currently are the ones widely used in the textile industry. Special reactive dyes for dyeing wood should be developed. Their structure should be established on the basis of quantum chemistry, and further study the competition mechanism between the hydrolysis reaction of the reactive dyes and the fixation reaction with wood, expecting a better dyeing effect of reactive dyes in wood dyeing.

3) Theoretical models about penetration, adsorption-diffusion of reactive dyes in accordance with reality have not been established. In the future theoretical research on Thermodynamics and kinetics in wood dyeing should be strengthened.

\section{Acknowledgements}

This paper is a part of the special project funded by the National Forestry Nonprofit Industry Foundation of China (ID: 201304509), and Students Research Learning and Innovation Experiment Program of Central South University of Forestry Science and Technology, The authors express our gratitudes.

\section{References}

[1] Li Y.Y., Wang J.J., Zhou Y., et al. Reactive dyes dyeing of the large cathay poplar veneer dyeing properties research [J]. Journal of central south forestry university, 2013, 33(4): 107-110.

[2] Li Y.F., Liu Y.X., Yu H.P., et al. Wood fluid permeation theory and research method [J]. Journal of forestry science, 2011, 47(2): 134-143.

[3] Lu L.C., Fan Y.M., Li M.F. High yield pulping of wood impregnated research progress[J]. Journal of Beijing forestry university, 2009, 31 (supp. L):210-215.

[4] Magnus T., Tapio H., Jarl B.R. Drift speed: a way of measureing diffusion and tortuosity of porous materials $[\mathrm{J}]$. Colloids and Surfaces A: Physicochemical and Engineering Aspects, 2001, 180: 23-31.

[5] Vicente C., Pedro C.. Estimating pure diffusion contributions in alkaline pulping processes[J]. Latin American Applied Research, 2001, 32: 151-159.

[6] Zanuttini M, Citroni M, Marzocchi V, et al. Pattern of alkali impregnation of poplar wood at moderate conditions[J]. Holzforschung, 2000, 54(6): 631-636.

[7] Kazi K M F, Gauvin H, Jollez P, et al. A diffusion model for the impregnation of lignocellulosic materials [J]. Journal of Technical Association of the Pulp and Paper Industry, 1997, 80(11): 209-219.

[8] Jiang X.J., Wang W.. Silk processing cotton reactive dyeing thermodynamics and dynamics research [J]. Journal of Shandong textile science and technology, 2013, (4):1-3. 
[9] Miao H.L.. Reactive dyes dyeing non-aqueous media and theoretical research [D]. Zhejiang university of technology, 2013.

[10] Sui S.Y., Zhu P., Xu C.H., et al. The research of dyeing kinetics properties of bamboo fiber [J] printing, 2006, (1): 11-15.

[11] Wang Y.G., Yue J.Q.. The research of poplar lateral diffusion of alkali impregnation [J]. Journal of nanjing forestry university (natural science edition), 2014, 38 (1) : 103-109.

[12] Fu Z.J.. The study on dyeing properties of polylactic acid fiber [D]. Wuhan university of technology, 2008.

[13] Li J.L., Yan C., Yu Z.C.. Dyeing properties and mechanism of cypress wood from the study [J]. Journal of zhejiang university of technology, 2010, 27 (4) : 534-539.

[14] Lv X.H.. Fast grown poplar color improved and dyeing mechanism research [D]. Central south forestry university, 2012.

[15] Yu H.H.. Hydrolysis and staining properties of mixed bifunctional reactive dyes [D], Dalian university of technology, 2010.

[16] Wang Z.J., Shao M., Shao J.Z. Vinyl sulfone reactive dyes hydrolysis kinetics study based on HPLC [J]. Journal of textile, 2006, 27 (9) : 9-13.

[17] Zhang R., Shen Y.F., Lin S.L., et al. Hydrolysis performance C.I. Reactive Blue 19 at low temperatures [J]. Journal of zhejiang university of technology, 2008, 25 (2) : 132-136.

[18] Olanrewaju O, Ige J, Omopariola S O. Alkaline hydrolysis of brilliant green in mixed cationic surfactant systems[J]. Central European Journal of Chemistry, 2011, 106-111. 